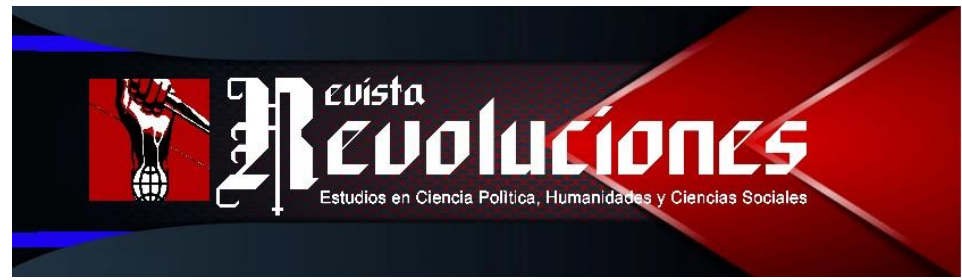

\title{
KACHKANIRAQMI: EL PENSAMIENTO SOCIAL ANDINO PARITARIO FRENTE AL PENSAMIENTO OCCIDENTAL INDIVIDUALISTA
}

\author{
Kachkaniraqmi: theparitarian andean social thought in the face of \\ individualistic western thought
}

\author{
Luis Alberto Añasco- \\ Huariccallo ${ }^{1}$ \\ UNIVERSIDAD NACIONAL DEL ALTIPLANO \\ PERÚ \\ luis.ahsociologo.17@gmail.com \\ https://orcid.org/oooo-0001-8284- \\ 1310
}

\author{
Dometila Mamani- \\ Jilaja ${ }^{2}$ \\ UNIVERSIDAD NACIONAL DEL \\ ALTIPLANO \\ PERÚ \\ domamani@unap.edu.pe \\ https://orcid.org/oooo- \\ ooo3-2357-8684
}

\author{
Humberto Mamani- \\ Coaquira 3 \\ UNIVERSIDAD NACIONAL DEL ALTIPLANO \\ PERÚ \\ hmamanic@unap.edu.pe \\ https://orcid.org/o0oo-0002- \\ 0569-860X
}

DOI: https://doi.org/10.35622/j.rr.2020.02.005

Recibido: 02-II-2020 / Aceptado: 28-VI-2020 / Actualizado: 21-I-2021

\section{Resumen}

El pensamiento Qapaq andino se ha transmitido de generación en generación en los ayllus, donde se mantiene modos de pensar, estilos de vida propios, diferenciada claramente de los modelos de vida individualistas occidental y eurocentrista. El objetivo del estudio consistió en analizar los términos del pensamiento Qapaq andino y hacer una diferenciación de los planteamientos occidentales. Bajo la metodología cualitativa se rastreó bibliográficamente los descriptos y se interpretó a los autores en referencia al fenómeno de la reivindicación indígena. Se evidenció que en los ayllus o comunidades campesinas originarias como de Sutuca Urinsaya y la comunidad Sutuca Anansaya, aún se mantiene vigente la práctica de los principios andinos del Allin Yachay o Wali Yatiña que significa saber bien, existe también el principio del Allin Munay o Wali Munaña traducido como querer bien y finalmente está el Allin Ruray o Wali Luraña traducido como el hacer bien para lograr el Sumaq Kawsay. Por tanto, esas manifestaciones en los diferentes trabajos y actividades cotidianas de los comuneros qhari-warmi o chacha-warmi que significa varón y mujer, sustentan la filosofía paritaria de los verdaderos habitantes de los pueblos invisibilizados del Perú Tahuantinsuyano.

\footnotetext{
${ }^{1}$ Licenciado en Sociología por la Universidad Nacional del Altiplano de Puno, Perú.

${ }^{2}$ Doctora en Educación, Magister en Ciencias de Educación con mención en educación en deportes, Licenciado en Educación Física, Licenciada en Educación Inicial, con estudios de Segunda especialidad en Educación primaria EBI, Segunda especialidad en Psicomotricidad (UNA-PUNO) primer puesto en estudios de pregrado, deportista destacada en fútbol femenino de la selección de estudiantes Puno, Docente de Pre y pos grado de la Facultad de Ciencias de la Educación de la Universidad Nacional del Altiplano Puno. Miembro del Instituto Universitario de Investigación Inudi Perú y Revista Innova Educación.

${ }^{3}$ Licenciado en Educación Secundaria y Magister en Investigación y Docencia Universitaria por la Universidad Nacional del Altiplano de Puno, Perú.
} 
Palabras Clave: Filosofía andina, descolonización, buen vivir, periferia, eurocentrismo.

\begin{abstract}
The andean Qapaq thought has been transmitted from generation to generation in the ayllus, where it maintains modes of thinking, own lifestyles, clearly differentiated from western and eurocentrist individualistic models of life. The objective of the study was to analyze the terms of andean Qapaq thinking and to differentiate western approaches. Under the qualitative methodology, the descriptions were traced bibliographically and the authors were interpreted in reference to the phenomenon of indigenous claim. It was evidenced that in the ayllus or native peasant communities such as Sucuca Urinsaya and the Sucuca Anansaya community, the practice of the andean principles of the Allin Yachay or Wali Yatiña, meaning to know well, is still in force. There is also the principle of the Allin Munay or Wali Munaña translated as wanting well and finally there is the Allin Ruray or Wali Luraña translated as doing well to achieve the Sumaq Kawsay. Therefore, these manifestations in the different daily work and activities of the communeros qhari-warmi or chacha-warmi, which means male and female, support the common philosophy of the true inhabitants of the invisibilized peoples of the Tahuantinsuyano Peru.
\end{abstract}

Keyword: Andean philosophy, decolonization, good living, periphery, eurocentrism.

\title{
INTRODUCCIÓN
}

Comenzar el análisis con la terminología noq'anchis con significado que hace referencia a los hijos del pueblo, llamados a desarrollar e investigar el pensamiento social andino de vital importancia en el proceso de descolonización de la cultura andina Tahuantinsuyana, ha sido y es aún uno de los mayores desafíos de los pueblos subalternos, ejemplo de ello es la educación oficial colonizadora y homogenizante, tanto en el nivel básico como en el superior. Sin embargo, ahora se considera que los quechuas, aymaras, urus y otras nacionalidades plantean la necesidad de fortalecer sus principios e intelectuales de la región. Por tanto, se reconoce el trabajo realizado por Gamaniel Churata (1957) reconocido por intelectuales por su trabajo el Pez de Oro, así mismo, están los escritos de Luduvico Bertonio (1612), quien realiza el Vocabulario de la Lengua Aymara.

En esa medida, se dice que el elemento descolonizador será la educación, ya que esta a diferencia de la educación occidental, educa no sólo para la razón, sino también para el corazón, lo que es esencialmente de relación complementaria (Huanacuni, 2015). Entonces, a partir de las investigaciones de diferentes intelectuales de la cultura originaria se expresa el pensamiento paritario o pensamiento Qhapaq4, como guía y timón del mundo andino (Lajo, 2005). Además, ejercer el pensamiento andino, diferenciando claramente de la occidental que es individualista

${ }_{4}^{4}$ Qhapaq. De acuerdo a los hablantes quechua y aymaras, significa guía, sabio, líder, elegido, poderoso, profeta.

Revista Revoluciones -61- Vol. 2, No 2 (2020), pp. 60-72

Esta obra está bajo una licencia internacional Creative Commons Atribución 4.0. 
y monocultural, obedece al Yanantin y Llapallanchis 5 que significa, ni machista ni feminista, sino todos juntos.

$\mathrm{Al}$ respecto, Mariátegui indicaba que la América india vive, es decir, los pueblos originarios aún están, pero el contexto central del problema del indio sigue siendo los medios de producción que subyace a la tierra o la Pachamama (Mariátegui, 2007). Entonces, superar los problemas desde los pensadores locales y propuestas endógenas al que se exponen los marginados, sigue siendo una alternativa positiva.

En esa lógica, para el proceso de descolonización iniciado por los grupos decoloniales de América Latina, resulta también interesante la propuesta local, por ello, uno de los exponentes puneños de la actualidad, Jesús Huanca-Arohuanca (2019a), considera que el camino hacia la revolución en los pueblos olvidados, es la reinvención de las ideologías y la misma perspectiva de la razón para crear una ontología diferente a la que se enseña en las universidades peruanas, es más, indica que en las aulas universitarias aún queda la praxis de la enseñanza del manual del comunismo ya en colapso. Por lo que, la mirada presente debe ser la epistemología propia, tal como De Sousa (2011) plantea cuando menciona que la vertiente del pensamiento crítico viene desde la misma periferia centrado en la lucha de clases dentro de los pueblos profundos. De manera que, priorizar el pensamiento endógeno bajo los principios andinos del Allin Yachay o Wali Yatiña que significa saber bien, del Allin Munay o Wali Munaña traducido como querer bien y el Allin Ruray o Wali Luraña traducido como el hacer bien, resulta necesario para lograr el Sumaq Kawsay (Lajo, 2010).

\section{METODOLOGÍA}

El trabajo de investigación se realizó bajo la metodología cualitativa acompañado del rastreo bibliográfico, revisión documental-argumentativa de diferentes fuentes de las bases de datos; Scopus, Scielo, Redalyc, Dialnet y OEI (Huanca-Arohuanca, 2019a; 2019b; 2020a; 2020b; 2020c; 2021; Huanca-Arohuanca, Canaza-Choque, \& Flores, 2020; Huanca-Arohuanca \& Geldrech, 2020; Huanca-Arohuanca \& Núñez, 2020; Huanca-Arohuanca \& Pilco, 2021; Tineo-Zaga et al., 2021). La técnica de observación se fundamentó en los cánones de los comuneros de Sutuca Hurinsay $a^{6}$ y Sutuca Hanansaya ${ }^{7}$ de la provincia de Lampa, ubicado en la región del altiplano de Puno. Todos ellos, mantienen el compromiso ético de seguir colaborando en las próximas investigaciones.

\section{DESARROLLO}

\section{Síntesis de la evolución rural en Puno}

${ }_{6}^{5}$ Llapallanchis. En español significa todos juntos y todos unidos.

${ }^{6}$ Sutuca Hurinsaya. Comunidad campesina quechua, jurisdicción de la provincia y distrito de Lampa ubicado en la parte baja.

7 Sutuca Hanansaya. Comunidad campesina quechua jurisdicción de la provincia y distrito de Lampa ubicado en la parte alta. 
En las comunidades campesinas de la región Puno, se conserva con nitidez el pensamiento andino paritario del cual todos y todas son parte. De manera que, resulta esencial el análisis del proceso de su evolución histórica. Para ello, se realizó un rastreo sobre a la población rural tal como se muestra en la tabla 1.

Tabla 1. Evolución de la población rural

\begin{tabular}{lccccccc}
\hline \multicolumn{7}{c}{ Evolución de la población censada rural, región Puno, 1940, 1961, 1972, 1981, 1993, 2007 y } \\
2017
\end{tabular}

Fuente: Instituto Nacional de Estadística e Informática - Censos Nacionales de Población y Vivienda, 1940, 1961, 1972, 1981, 1993, 2007 y 2017.

Se aprecia claramente que la población rural de 1940 a 2017, sufrió un ligero cambio constituido en más de 64757 (INEI, 2018), es decir, esas poblaciones rurales de la región Puno aún mantienen sus costumbres, tradiciones, cultura y modos de pensar conocidos por los intelectuales actuales como, filosofía andina. Con relación al idioma o lengua que aprendieron a hablar en su niñez, las personas de cinco y más años de edad, destacan el quechua en 42,9\%, el castellano en $28,0 \%$ y el aimara en $27,0 \%$. Con referencia sobre la auto identificación étnica, la población mayor de doce años se consideran quechua es de 537972 y el aimara es de 318 363, quienes en conjunto representan al 90,7\% de dicho grupo etario (INEI, 2018). De manera que, la presencia de los quechuas y aimaras se mantiene vigente, sin embargo, la población Uro, a pesar de ser escaso en las islas flotantes, aún persiste revalorando su cultura en el Lago Titicaca.

\section{Qori qelqa en el templo de Koricancha}

De acuerdo al estudio realizado por Javier Lajo (2005), en el templo del Urin Qosqo $^{8}$ o Koricancha ${ }^{9}$, destruido por los sacerdotes cristianos, la memoria histórica representado de forma simbólica con el patio principal del Templo Mayor, todavía se conserva. De acuerdo a la figura 1, en el Qori cancha se visualiza la traducción del Runa simi $i^{10}$, Qori $q e l q{ }^{11}$, el Inti ${ }^{12}$ y la Quilla $^{13}$, todos ellos constituidos en referencia a una pareja qhari ${ }^{14}-$ warmi $^{5}{ }^{50}$ en la versión aimara-quechua, chach ${ }^{16}$ - warmi, es decir, la dualidad que se expone puede hacer referencia a la inexistencia del machismo y el feminismo ya que en la cultura ancestral no existe tal situación.

${ }^{8}$ Urin Qosqo. Parte baja de la ciudadela del Cusco.

${ }_{9}^{9}$ Koricancha. Lugar sagrado conocido como templo de oro.

1o Runa Simi. Categoría quechua, en español idioma del varón y la mujer.

${ }^{11}$ Qori qelqa. Escritura en lámina de oro.

12 Inti. Dios Sol que representa al varón.

${ }_{13}$ Quilla. Diosa Luna que representa a la mujer.

${ }^{14}$ Qhari. Categoría quechua, en español significa varón.

${ }^{15}$ Warmi. Categoría quechua y aimara, en español se denomina mujer.

${ }^{16}$ Chacha. Categoría aimara que significa varón. 
Para Brechetti (2003), el pensamiento y la vida religiosa de los incas, como también de la población andina, se representan por la estructura dual, de la parte de arriba-abajo, derechaizquierda, masculino-femenino, una filosofía netamente andina que hoy todavía se encuentra viva en las tradiciones y fiestas de los campesinos quechuas y aimaras. En las comunidades estudiadas de Lampa, el varón y la mujer cumplen un rol opuesto pero complementario, por ejemplo, en los trabajos agrícolas de sembrío de papa, la mujer realiza el iluy ${ }^{17}$ y el varón a través de su fuerza realiza la tarea del tarpuy ${ }^{18}$, pero todas las labores agrícolas se realizan de manera conjunta y recíproca.

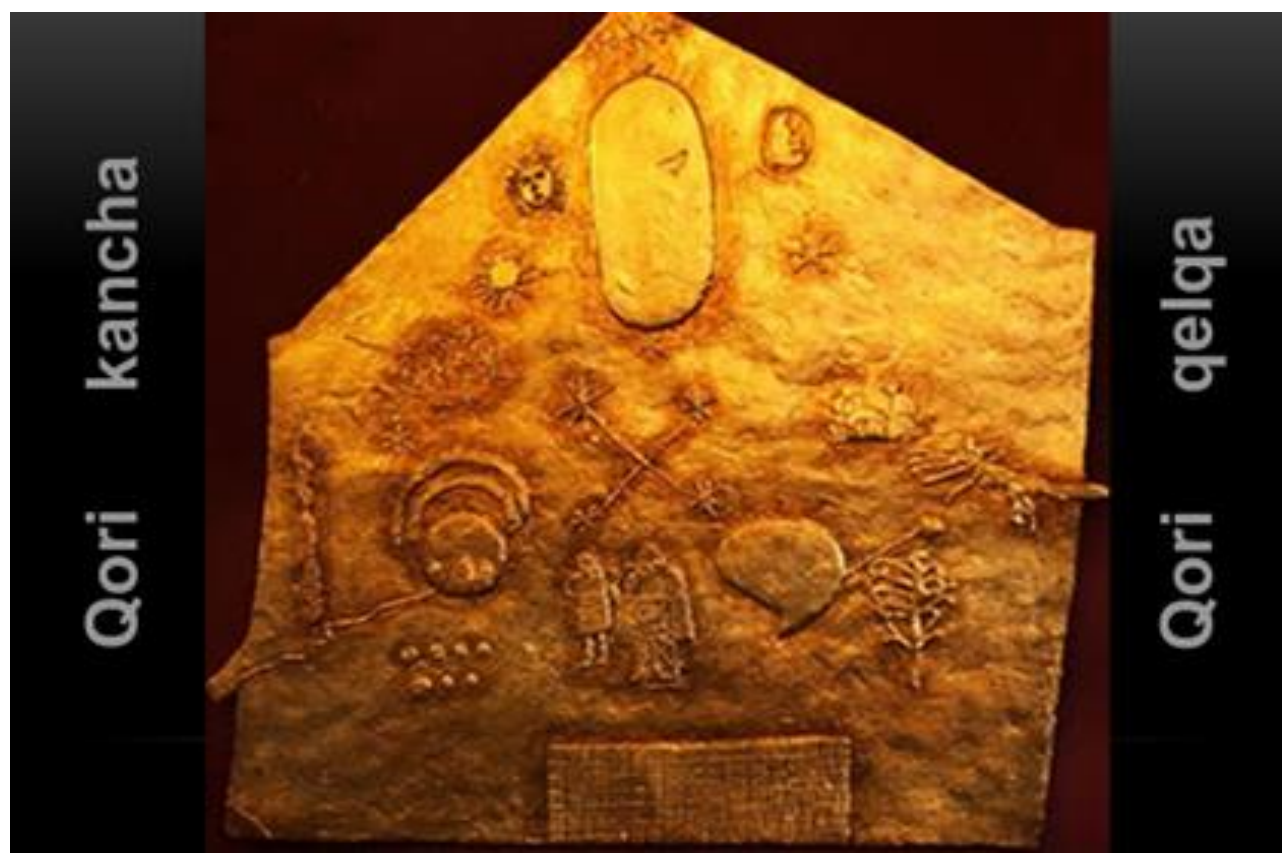

Figura 1. Lámina de oro en el Qori cancha (Google, 2020).

Además de lo que ya se describió, en la imagen aparece también cuatro estrellas orientadas al norte-sur, este-oeste y una estrella central en el medio de la intersección. En la parte superior aparece el Yaya Inti y Mama Quilla, por lo que en términos andinos significa Hanan Pacha o espacio que solo ocupa el Pachatata como padre cósmico del tiempo-espacio. Así mismo, en los escritos de Huanacuni (2010) un claro ejemplo son las naciones aimaras y quechuas que conciben los términos anteriores de la Pachakama o Pachatata ${ }^{19}$ y la Pachamama $^{20}$ generadora de toda forma existencial en el mundo. Entonces, las categorías a priori expresan un profundo significado de la forma de vida que tienen los andinos y la capacidad de leer el tiempo, sus significados, el comportamiento de los seres vivos, ya que en la memoria genealógica está relacionado con la madre naturaleza y por ello los Yachaq realizan ofrendas en diferentes momentos del año, pero siempre teniendo presente la dualidad, es decir, dos hojas de coca a más, pero par.

17 Iluy. Siembra de papa, colocar la semilla de la papa en los surcos.

18 Tarpuy. Siembra de papa, usar la chaqui taqlla para apertura y colocar la papa.

19 Pachatata. En español significa padre cosmos, al mismo tiempo representa al mundo externo y el varón.

${ }_{20}$ Pachamama. En español es madre tierra, madre naturaleza, representa a los sentimientos y la mujer. 


\section{Cosmovisión andina visto desde el altiplano}

En el mundo andino, existe una milenaria forma de entender el cosmos mediante la observación, puesto que la cosmovisión vincula al hombre andino con el universo total (Milla, 2018). De manera que, bajo la interpretación del pacco $^{21}$, yachaq ${ }^{22}$, yatiri ${ }^{23}$, el jawa pacha ${ }^{24 y}$ anqha pacha ${ }^{25}$, tienen una relación intrínseca con las constelaciones del cual se saca mucha información para el conocimiento de los sabios andinos. Así mismo, existe el $u k h u$ pacha ${ }^{26} \mathrm{y}$ el manqha pacha ${ }^{27}$, de los cuales fluyen los sentimientos, las emociones y las vivencias del todos los humanos vinculados a los andes, es decir, el tiempo es pacha $k u t i^{28} \mathrm{y}$ muchas veces, el tiempo para los que manejan la cosmovisión andina está de vuelta, lo que se categoriza como el pacha muyu ${ }^{29}$. En educación, por ejemplo, no solo el maestro enseña a los estudiantes, sino que el niño también le enseña al maestro; le enseña su alegría, su inocencia, su actuar sin temor, sin estructuras, una educación, de ida y de vuelta, donde ante todo se comparte la vida (Huanacuni, 2015).

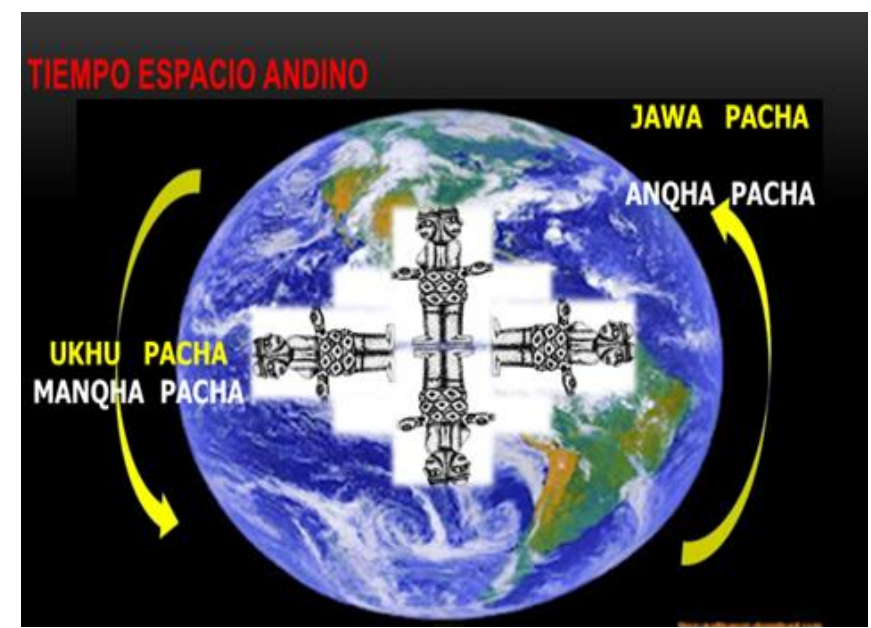

Figura 2. El tiempo y espacio andino como interpretación cíclica.

Fuente: tomado de Huanacuni (2010).

Según Zenteno (2009), el término pacha es la unión de dos energías, ya que pa proviene de la expresión paya que significa dos y cha viene de chama con significado de fuerza. La esencia del cosmos es la naturaleza, porque está estructurado por opuestos complementarios. Así mismo, para la cosmovisión de los pueblos indígenas originarios, primero está la vida en

${ }^{21}$ Pacco. En idioma puquina se conceptualiza como sabio al recibir la energía del rayo.

22 Yachaq. En idioma quechua el significado asignado es sabio, al recibir la energía del rayo.

23 Yatiri. En idioma aimara significa sabio, al recibir la energía del rayo.

24 Jawa Pacha. Categoría quechua que en español se conoce como mundo externo del cual se adquiere conocimientos.

${ }^{25}$ Anqha Pacha. Categoría aimara que en español significa mundo externo del cual se adquiere conocimientos.

${ }^{26}$ Ukhu Pacha. Categoría quechua en español es conocido como mundo interior, del cual nace los sentimientos del runa.

${ }_{27}^{27}$ Manqha Pacha. Categoría aimara que se conoce como mundo interior, del cual nace los sentimientos del jaqe.

${ }^{28}$ Pacha Kuti. Se aprecia como el movimiento de rotación de la tierra.

${ }_{29}^{2}$ Pacha Muyu. En español significa el movimiento de traslación de la tierra. 
relación armónica y equilibrada, por lo que qamaña se aplica a quienes saben vivir. Ahora bien, el término de suma qamaña se traduce como vivir bien (Huanacuni, 2015). Por consiguiente, los elementos esenciales de la cosmovisión andina con respecto al bienestar social es el intercambio del todo, que está conectado de una forma u otra (Cruz, 2018), el pensamiento andino, todos los procesos y fenómenos naturales como la crianza de la vida. Como tales, deben desarrollarse en armonía y en su conjunto constituyen la armonía de la Pachamama (Van Kessel \& Enríquez, 2002), es decir, sin la madre naturaleza no hay vida.

\section{Principios para el sendero del Sumaq Kawsay}

Los principios andinos constituidos como; el Allin Yachay o Wali Yatiña - saber bien, el Allin Munay o Wali Munaña - querer bien, el Allin Ruray o Wali Luraña - hacer bien y el Allin Kay o Wali Jakaña - estar bien, están interconectados para encaminar el Sumaq Kawsay o Suma Qamaña - vivir bien entre todos nosotros (Lajo, 2010). A diferencia del pensar occidental, como se aprecia en lo descrito, existen formas de recrear la comunidad y mantener la reciprocidad que conlleva a sociedades igualitarias.

Para Huahuasoncco (2013), yanantin es la primera ley del pensamiento Qapaq, yana significa templado, enamorado cautivo de amor, sea varón o mujer. Tinkuy es la segunda ley del pensamiento Qapaq, puesto que es el método o vinculo de proporcionalidad de figuras complementarios del cuadrado y el circulo para finalmente constituir el yanantincuy conocido a la vez como pareja primordial. En cambio, el Ayni3o y el Mink' $a^{31}$ representan en todos los pueblos de la periferia el principio de reciprocidad y complementariedad, máximas que hasta la actualidad están vigentes.

${ }^{30}$ Ayni. Categoría quechua que en español representa la reciprocidad del hoy por mi mañana por ti.

${ }^{31}$ Mink'a. categoría quechua en español complemento. 


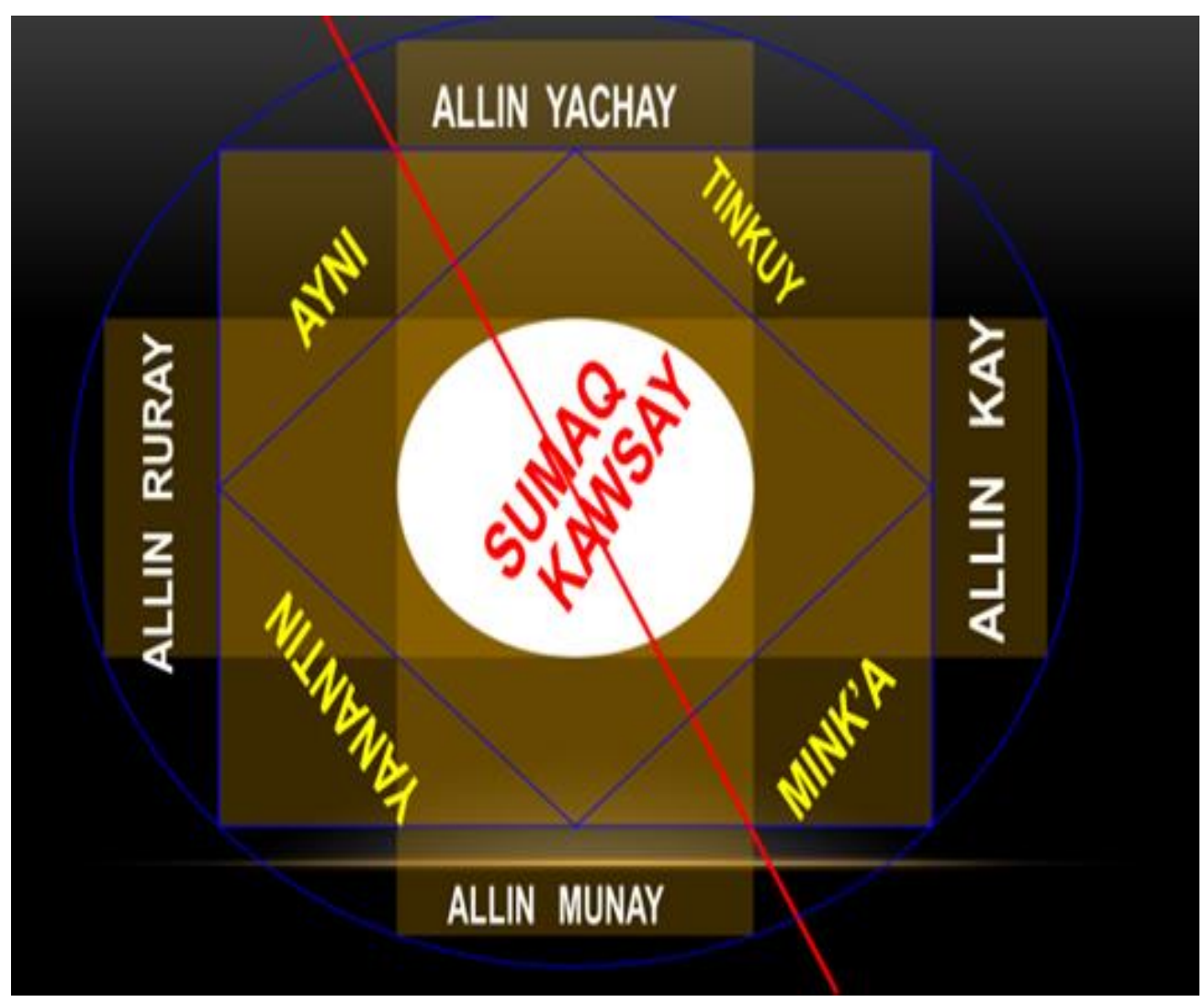

Figura 3. Principios del pensamiento Qapaq. Fuente: tomado de Lajo (2005).

\section{Respuestas a las ideologías occidentales}

El pensamiento occidental nace con los griegos, específicamente con los filósofos; Sócrates, Platón y Aristóteles. En ese criterio. los primeros planteamientos sociopolíticos de la educación fueron de mayor influencia en el mundo griego y para la cultura occidental. A partir de ahí es donde se formularon las principales interrogantes de orden filosófico y ético como: ¿cuál será lo mejor para el hombre? y ¿cómo vivir plenamente y feliz? Por lo que la educación debía formar al hombre griego (Morales, Bermudez, \& García 2018) bajo una racionalidad ontológicamente situado, tal como se pretende hoy.

Parece claro a estas alturas, la humanidad ha entendido a través de sus intelectuales que el amor a la sabiduría en términos etimológicos solo significa dominar y despojar a los que no poseen lo mencionado. Pareciera que cuanto más se sabe, más se puede y más se domina a los otros seres. Quizás la idea de que siempre existirán los medios de dominación constituidos por diversas maneras en todo el proceso civilizatorio (Huanca-Arohuanca, Canaza-Choque, Escobar-Mamani, et al., 2020), sea una condición fatal. Al respecto el filósofo holandés Josef Estermann (2009) considera que la concepción occidental es dominante de las otras filosofías derivadas a priori, porque no pueden existir filosofías africanas e indígenas lado a lado con la occidental, ni filosofías proccidentales (prehispánicas, prelusitanas y precoloniales). 
En efecto, en el sistema educativo, todo el constructo teórico y sistémico está apegado a esa idea, tanto es así que los sectores rurales que antes practicaban por ejemplo el idioma ancestral o la identidad cultural con contenidos del pensamiento paritario han dejado secuencialmente hacerlo. A pesar de todo lo que presenta el sistema educativo, en el sector rural aimara, el nivel de la identidad cultural en los estudiantes sigue siendo significativo, ya que, en la escala cualitativa Si poseen identidad cultural, se encuentran al 58\% (HuancaArohuanca, Sucari, et al., 2020).

\section{Respuesta al liberalismo}

Al pensador Jhon Locke se le conoce como el padre del liberalismo por sostener que todo gobierno surge de un pacto o contrato revocable entre individuos, con el propósito de proteger la vida, la libertad y la propiedad de las personas (Vánagy, 2000). Para Montesquieu, la libertad consiste en el pacífico disfrute de los bienes que tiene cada ciudadano (Giner, 2001). Mientras tanto, para Jean Baptiste Colbert (1619-1683), quien fue ministro de Hacienda de Luis XIV, sentenció a la humanidad con su famosa frase; laissez faire et laissez passer; le monde va de lui méme (dejar hacer y dejar pasar, el mundo marcha por sí mismo), sentencia que estaría sujeta a una patológica economía mundial.

Así mismo, Adam Smith, David Ricardo y Thomas Robert Malthus, todos ellos economistas ingleses, proponen el desarrollo económico basado en el libre mercado (Vargas, 2007). Esos modelos desarrollados han evolucionado, ahora se les denomina sistema neoliberal, modelo de crecimiento económico consumista como sinónimo de desarrollo que ha entendido al bienestar humano como la capacidad de consumo, medida por el incremento de ingresos económicos (Durán, 2011), que sin lugar a dudas, está en un proceso de colapso.

En el pensamiento occidental, esa facultad se limita al modo humano y personal de vivir; en cambio para el pensamiento andino, cada cosa tiene su carácter personal y se comunica con otros seres (Van Kessel, 2003). Visualizado desde la educación occidental, se tiene como máxima la búsqueda del conocimiento, la competitividad, calidad total, sin embargo, en términos de la filosofía andina, el pensamiento crítico es la reflexión situada y meditada para lograr el Suma Qamaña. En ese contexto, los aimaras y quechuas del altiplano, buscan establecer una educación emancipadora que pueda garantizar la libertad como herramienta fundamental de la democracia (Huanca-Arohuanca \& Canaza-Choque, 2019; Canaza-Choque \& Huanca-Arohuanca, 2018).

Por tanto, en las comunidades originarias, la sabiduría andina siempre buscará la armonía entre todos los seres vivientes, entre hermanos y hermanas, ya que como hijos de la Pachamama y el Pachatata, todos habitan bajo los estándares del Wawqekuna32Panakuna 33 , es decir, todos son hermanos y hermanas en el mundo andino. De modo que, la práctica de la reciprocidad-ayni y la complementariedad-mink'a, son esenciales. Claro

${ }^{32}$ Wawqekuna. Categoría quechua que hace referencia a hermanos.

33 Panakuna. En español representa a la denominación de hermanas. 
ejemplo de ello es la Guerra con Chile, donde varones y mujeres cumplieron un rol importante para enfrentar a un enemigo que tenía toda la ventaja (Huanca-Arohuanca et al., 2019; Huanca-Arohuanca, 2021), a pesar de la penosa realidad, tuvieron que luchar hasta morir por la patria.

\section{Respuesta al socialismo o comunismo}

El otro extremo se encuentra la corriente occidental denominado socialismo científico evocada por Marx y Engels (1848), por lo que en sus escritos del manifiesto comunista, indica que la historia de todas las sociedades hasta nuestros días es la lucha de clases en el cual se enfrentan; hombres libres y esclavos, patricios y plebeyos, señores y siervos, maestros y oficiales, en una palabra; opresores y oprimidos se enfrentaron siempre, mantuvieron una lucha constante, velada unas veces y otras francas y abiertas. Por tanto, la irrupción de las tesis de la filosofía de la praxis, fundamentadas en el historicismo es la forma de pensar que ubica al hombre como el eje de la vida, de la historia y la política (Cortés, 2019).

Al respecto, Anibal Quijano (2014) sostiene que la colonialidad del poder se basa en la imposición de la idea de raza como instrumento de dominación, siendo en todas las latitudes un factor limitante de los procesos de construcción del Estado-nación, puesto que, todos ellos están basados en el modelo eurocéntrico que se logra imponiendo el modelo europeo alrededor de las relaciones coloniales (Quijano, 2014; Alanoca, 2019).

En ese criterio, una concepción sobre democracia desde lo exterior, anula el normal desarrollo de los pueblos, por lo que, la función de los Estados es elemental. Como se sabe, todos los ciudadanos tienen los mismos derechos y todos los habitantes son catalogados igualitariamente. Es más, las democracias modernas son consideradas como liberales, cuando a ese primer principio fundamental se suma un segundo: la libertad de los individuos (Zamitiz, 2018). Por otro lado, en los estudios de Habermas, la fase final de evolución, es una sociedad racional. Aquí la racionalidad supone la supresión de las barreras que perturban la comunicación (Ritzer, 1997).

Sea cual fuese la realidad, todos los pueblos subalternos están llamados a la revolución para alcanzar la descolonialidad epistemológica, cultural y del poder. Todo lo a priori implica el cuestionamiento de los supuestos sobre los que se construyeron las estructuras del saber eurocéntricas y el señalamiento de las hipótesis alternativas, para que finalmente los conocimientos que provienen de las prácticas de los pueblos indígenas se pongan en marcha y que el Sumak Kausay o Suma Qamaña sea lo que prevalezca.

\section{CONCLUSIONES}

Como se aprecia en el estudio, existen claras diferencias entre el pensamiento andino y el pensamiento occidental, en tal sentido, se tiene que indicar que en las comunidades analizadas aún se desarrolla la sabiduría andina de tendencia paritaria, un conocimiento que se heredó de

Revista Revoluciones -69- Vol. 2, No 2 (2020), pp. 60-72

Esta obra está bajo una licencia internacional Creative Commons Atribución 4.0. 
los Qolla Suyos que tenían como gobierno al Pachatata circunscrito como el Qhapaq Qolla y la Pachamama conceptualizado en la Qhapaq Qoya, todos ellos, gobernaron con la sabiduría dualista que posteriormente se materializó en la confederación del Tawantinsuyo (Huahuasoncco, 2013). Lo anterior responde a la sabiduría ancestral de la dualidad, en otras palabras, a pesar de que podrían ser opuestos, siempre existe el principio de complementariedad conocido en el idioma quechua como masachakuy o vivir en pareja. Un ejemplo claro es el huk qhariwan huk warmiwan tiyapuy que significa contraer matrimonio entre varón y mujer, pero de ninguna manera existe la unión de varón y varón o mujer y mujer, si eso fuera el caso, ya no existiría lo opuesto y complementario, asimismo, los yachaq indican que todo lo descrito se aprendió de las wallatas que se aman hasta la muerte pese a las dificultades.

El pensamiento occidental y su peculiar estilo de vida, se conceptualiza en la búsqueda de la racionalidad, la competitividad y calidad total, fomentando el individualismo y el machismofeminismo supuestamente para solo vivir un poco mejor que los demás. El hombre occidental prefiere lo general, porque eso le acerca al conocimiento de las leyes o regularidades universales que le permiten el control, el dominio y hasta la manipulación de la realidad. El hombre andino en cambio, busca más bien la convivencia con la naturaleza y la inmersión en su seno como fuente de vida y renovación. Es decir, la cultura se manifiesta siempre con sus opuestos complementarios, día-noche, amanecer-atardecer, varón-mujer y calor-frío.

Por tanto, esas manifestaciones en los diferentes trabajos y actividades cotidianas de los comuneros qhari-warmi o chacha-warmi con significado de varón y mujer, sustentan la filosofía paritaria de los verdaderos habitantes de los pueblos invisibilizados del Perú Tahuantinsuyano. Además, es una lección para el mundo que en esas comunidades aún se practiquen costumbres propias, a pesar de que la población joven de esas comunidades esté en proceso de alienación referente a su identidad cultural y sus propios valores.

\section{REFERENCIAS BIBLIOGRÁFICAS}

Alanoca-Arocutipa, V. (2019). Desde las voces silenciadas en el surandino, resurgen nuevas líneas de pensamiento emancipatorio. Revista Revoluciones, 1(1), 14-24. http://revistarevoluciones.com/index.php/rr/article/view/3

Bertonio, L. (1612). Vocabulario de la lengua aymara. Francisco del Canto.

Brechetti, A. (2003). “...Los pintaré como estaban puestos hasta que entró a este reyno el santo Ebangeleo" Santacruz Pachacuti Yamqui, 1613. Anales del Museo de América. 11, 81-102. https://dialnet.unirioja.es/servlet/articulo?codigo $=961567$

Canaza-Choque, F. A., \& Huanca-Arohuanca, J. W. (2018). Perú 2018: hacia una Educación Intercultural Bilingüe sentipensante. Sciendo. Ciencia Para El Desarrollo, 21(4), 515522. https://doi.org/10.17268/sciendo.2018.058

Churata, G. (1957). El pez de oro. Canota.

Cortés, D. (2019). Neo marxismo y revolución cultural. Utopía y Praxis Latinoamericana, 24, 149-166. https://dialnet.unirioja.es/servlet/articulo?codigo $=7527675$

Cruz, M. (2018). Cosmovisión andina e interculturalidad: Una mirada al desarrollo sostenible 
ISSN: 2710-0499 ISSN-L: 2710-0480

desde el Sumak Kawsay. Chakiñan, 5, 119-132.

http://scielo.senescyt.gob.ec/pdf/rchakin/n5/2550-6722-rchakin-05-00119.pdf

Durán, M. (2011). Sumak Kawsay o Buen Vivir, desde la cosmovisión andina hacia la ética de

la sustentabilidad. Pensamiento Actual, 1O(14), 51-61.

https://revistas.ucr.ac.cr/index.php/pensamiento-actual/article/view/3746/3622

Estermann, J. (2009). Filosofia andina sabiduria andina para un mundo nuevo. ISEAT Bolivia.

Giner, S. (2001). Teoría sociológica clásica. Ariel S.A.

Huahuasoncco, J. (2013). Pensamiento Qhapaq Qolla. In Qollasuyo. Editorial Nawpas.

Huanacuni, F. (2010). Buen vivir/Vivir bien. CAOI.

Huanacuni, F. (2015). Educación comunitaria. Integra Educativa, 7(4), 159-168.

http://www.scielo.org.bo/pdf/rieiii/v8n1/v8n1_ao8.pdf

Huanca-Arohuanca, J. W. (2019a). El despertar de una nueva era: colonización y camino hacia la revolución. Revista Revoluciones, 1(1), 1-3. http://revistarevoluciones.com/index.php/rr/article/view/1/2

Huanca-Arohuanca, J. W. (2019b). El discurso filosófico y la violencia política en la Nación Aymara - Ácora [Universidad Nacional del Altiplano]. http://repositorio.unap.edu.pe/bitstream/handle/UNAP/12758/Huanca_Arohuanca_ Jesús_Wiliam.pdf?sequence $=1$ \&isAllowed $=\mathrm{y}$

Huanca-Arohuanca, J. W. (2020a). Caleidoscopio social al Covid-19: pánico y desesperación en tiempos de aislamiento. Revista Universidad y Sociedad, 12(6), 226-231. https://rus.ucf.edu.cu/index.php/rus/article/view/1836

Huanca-Arohuanca, J. W. (2020b). Contrahegemonía y la lucha por la educación en el sur del Perú. Editorial Académica Española.

Huanca-Arohuanca, J. W. (2020c). Retropías y distopías de la educación en Puno. Revista Helios, 4(1), 270-271. https://doi.org/10.22497/Helios.41.4116

Huanca-Arohuanca, J. W. (2021). Narrativas de guerra y resistencia: participación de la mujer austral del Perú en la Guerra del Pacífico. Encuentros. Revista de Ciencias Humanas, Teoría Social y Pensamiento Crítico, 13, 50-59. https://doi.org/http://doi.org/10.5281/zenodo.4395218

Huanca-Arohuanca, J. W., \& Canaza-Choque, F. A. (2019). Puno: Educación rural y pensamiento crítico. Hacia una educación inclusiva. Revista Helios, 3(1), 97-108. https://doi.org/10.22497/Helios.31.3106

Huanca-Arohuanca, J. W., Canaza-Choque, F. A., Escobar-Mamani, F., \& Ruelas, D. (2020). En defensa del pluralismo latinoamericano: las esferas de la justicia y la igualdad compleja en Michael Walzer. Un dilema pendiente por atender. Chakiñan. Revista de Ciencias Sociales y Humanidades, 11, 92-103. https://doi.org/10.37135/chk.002.11.07 Huanca-Arohuanca, J. W., Canaza-Choque, F. A., \& Flores, E. (2020). El dolor de los subalternos y el deseo de una revolución inconclusa: Narrativas sobre la violencia política en la Nación Aymara - Perú.Comuni@cción: Revista De Investigación En Comunicación $Y$ Desarrollo, 11(2), 177-189. https://doi.org/10.33595/22261478.11.2.436

Huanca-Arohuanca, J. W., \& Geldrech, P. (2020). Planificación educativa y gestión 
pedagógica-estratégica-operacional en las instituciones del nivel inicial en el sur del Perú. Revista Conrado, 16(76), 369-376. https://conrado.ucf.edu.cu/index.php/conrado/article/view/1497

Huanca-Arohuanca, J. W., \& Núñez, L. (2020). Estimaciones y contrastes de la pandemia en

Perú y en el contexto mundial. Educare Et Comunicare. Revista Científica de La Fcultad de Humanidades, 8(2), 10-20. https://doi.org/10.35383/educare.v8i2.440

Huanca-Arohuanca, J. W., \& Pilco, N. (2021). Acciones revolucionarias en Ámérica Latina: Puno y el Alto Perú durante el proceso de independencia (1809-1825). Chakiñan. Revista de Ciencias Sociales y 14 Humanidades, https://doi.org/10.1590/SciELOPreprints.1364

Huanca-Arohuanca, J. W., Sucari, W. G., Zapana, R. A., \& Huarancca, R. A. (2019). Construyendo la memoria histórica: participación de la mujer puneña en la Guerra con Chile. Revista Revoluciones, $1(1), \quad 4-13$. http://revistarevoluciones.com/index.php/rr/article/view/2

Huanca-Arohuanca, J. W., Sucari, W., Huayanca, P., Yabar, P., Ticona, E., \& Supo, F. (2020). Identidad atávica en los estudiantes bilingües del espacio rural en el Altiplano Aymara de Ilave. Revista Innova Educación, 2(1), 114-127. https://doi.org/10.35622/j.rie.2020.01.013

INEI. (2018a). Censos 2017: Departamento de Puno tiene 1172697 habitantes. INEI.

INEI. (2018b). III Censo de comuidades nativas 2017.

Lajo, J. (2005). QHapaq ñan: La ruta inka de la sabiduria. Amaro Runa.

Lajo, J. (2010). Qhapaq Ñan: La escuela de sabiduría andina. Amaro Runa.

Mariátegui, C. (2007). 7 ensayos de la interpretacion de la realidad peruana. Fundación Biblioteca Ayacucho.

Marx, C., \& Engels, F. (2019). Manifiesto del partido Comunista. (ed. 19). Alianza Editorial, S. A.

Milla, C. (2018). Cultura e identidad en los países andinos. Chakiñan, 27-36. http://scielo.senescyt.gob.ec/pdf/rchakin/n6/2550-6722-rchakin-o6-00027.pdf

Morales, P., Bermudez, J., \& García, J. (2018). Planteamientos sociopolíticos de la educación en el pensamiento filosófico griego antiguo : Sócrates, Platón y Aristóteles. Innova Research Journal $3(2)$, $136-146$. https://dialnet.unirioja.es/servlet/articulo?codigo $=6324898$

Quijano, A. (2014). Colonialidad del poder, eurocentrismo y América Latina. CLACSO, 777832.

Ritzer, G. (1997). Teoría Sociológica contemporanea. McGraw.

Sousa, B. (2015). Una epistemologia del sur. Editorial Siglo XXI.

Tineo-Zaga, Y., Casa-Coila, M. D., \& Huanca-Arohuanca, J. W. (2021). Gestión pedagógica y cultura organizacional en la Institución Educativa Andrés Bello de Yunguyo , Perú. $\begin{array}{lllll}\text { Educación } & y & \text { Sociedad, } & 19(1), & 153-169 .\end{array}$ http://revistas.unica.cu/index.php/edusoc/article/view/1695

Van Kessel, J. (2003). La economía andina de crianza; actores meta-economicos. Revista de Ciencias Sociales, 13, 66-73. https://www.redalyc.org/pdf/708/70801306.pdf

Van Kessel, J., \& Enríquez, P. (2002). Señas y señaleros de la santa tierra agronomia andina. Abya Yala, Quito. 
Vánagy, T. (2000). Pensamiento politico de Jhon Locke y el surgimento del liberalismo. CLACSO, 74-76.

Vargas, J. (2007). Liberalismo, Neoliberalismo, Postneoliberalismo. Revista Mad, 17, 66-89. http://www.facso.uchile.cl/publicaciones/mad/17/vargas_04.pdf

Zamitiz, H. (2018). Dela de la democracia liberal a la posdemocracia: explicaciones sobre malestar ciudadano contra las elecciones. Estudios Politicos, 9, 27-55. http://www.scielo.org.mx/pdf/ep/n45/0185-1616-ep-45-27.pdf

Zenteno, H. (2009). Acercamiento a la visión cósmica del mundo Andino. Puno Cero, 14(18), 83-87. http://www.scielo.org.bo/pdf/rpc/v14n18/v14n18a10.pdf 